\title{
A Survey Paper on Crater Detection
}

\author{
Jyoti Patil ${ }^{1}$, Dr. Srinivas Narasimha Kini ${ }^{2}$ \\ ${ }^{1}$ M.E (Computer) Department of Computer Engineering, Jayawantrao Sawant College of Engineering, Pune, India. \\ Savitribai Phule Pune University, Pune, Maharashtra, India -411028 \\ ${ }^{2}$ Professor (Computer) Department of Computer Engineering, Jayawantrao Sawant College of Engineering, Pune, India. \\ Savitribai Phule Pune University, Pune, Maharashtra, India -411007
}

\begin{abstract}
Craters are the most plenteous landform on the planet surface, which could give key intimations to planetary science. Because of varieties in the landscape, brightening, and scale, it is trying to distinguish Craters through remote detecting pictures and it requires an effective crater feature extraction method. In this paper, we address this issue utilizing Gist features, which can give exceedingly compelling depictions on crater's nearby edges and worldwide structure. The proposed crater's discovery technique contains three key steps. To begin with, we separate all candidate craters on a planet picture utilizing edge based technique and Hough transform. Second, Gist elements are created from chose preparing tests. Third, Craters discovery is led utilizing Gist features vectors with random forest classification. Contrasted with pixel based also, Haar-like components, our strategy indicates more exact craters acknowledgment, and accomplishes fulfilled results in the analyses directed on the Mars Orbiter Camera (MOC) database.
\end{abstract}

Keywords: Craters Detection, Gist features, Random forest, Hough transform, Haar Like Elements.

\section{Introduction}

Impact Craters are among the most studied features on Mars. They are structures formed by collisions of meteoroids with the Martian surface. Their importance stems from the wealth of information that detailed analysis of their distributions and morphology can bring forth. For example, statistics of crater sizes forms the basis for geological stratigraphy of Mars. In addition, knowledge of crater morphologies enables studies of a number of outstanding issues in Martian geomorphology, such as: the nature of degradation processes, regional variations in geologic material, and distribution of subsurface volatiles. Thus, surveying Martian craters is an important task in planetary research. What's more, Crater are perfect route historic points, which are utilized for exact movement estimation for landing control arrangement of planetary tests, for example, landscape relative route. Consequently, checking and measuring the craters on the planet of solar framework is an imperative work for planet science. Be that as it may, with vast volumes of picture information gained by tests of Moon, Mars, and different planets, manual pit recognition, index, and investigation have turned into a testing and tedious undertaking [2]. Along these lines, we require effective and dependable programmed crater discovery strategies.

Numbers of crater detection algorithms (CDAs) have been proposed over the last few decades to address the challenges involved [3].Today, as in the past, efficient crater detection in planetary images remains as a daunting task due to the following challenges. Challenge 1: Lack of distinguishing features. Craters, as a landform formation, lack strong common features distinguishing them from other landform formations. Their sizes differ by orders of magnitude. Their rims have often been eroded since their formation millions of years ago, resulting in shapes that depart significantly from circles. They frequently overlap, complicating the task of their separation from background. Challenge 2: Heterogeneous morphology in images. Planetary surfaces are not homogeneous where non uniform surface morphology frequently exhibits. Furthermore, planetary images may be taken at different lighting conditions, at different resolutions, and their quality varies so that even morphologically identical craters may have different appearances in different images. Challenge 3: Huge amount of sub kilometer craters in high-resolution planetary images. The size distribution of craters follows power-law; large craters that can be easily identified manually are rare and small sub kilometer craters are abundant.

Highlight extraction for crater picture representations is a key procedure, and it is firmly identified with crater recognition results. Great components ought to give crucial portrayal of the subject, catch the discriminative data, and offer a decent execution in pit identification classifier [6]. For crater pictures, an proficient element extraction strategy ought to be powerful in diverse scales, brightening points, and landscapes. Numerous components have been created, for example, standardized pixel-based elements [1], Haar-like elements [7], [8], and other neighborhood descriptors [9], [10]. For pixel-based element, all the first pixels are standardized to dispense with the impact of splendor and differentiation on unique crater pictures by a complexity standardization method. At that point, we acquire the pixelbased component by changing over the standardized dark picture lattice into one-dimensional vector. Pixel-based components keep the first picture data, however they can be delicate to area, landscapes condition, and some other components which influence the picture nature of the planetary surface [11]. For Haar-like components, almost 14 000 elements are acquired by testing every picture hinder with an arrangement of ternary veils, took after by an element determination technique [12]. They are normal to speak to the inborn components to particular the holes and non craters [13]. In this manner, we adjust the Gist components to recognize craters on Martian remote detecting pictures in this paper. The Gist was at first proposed by Potter and Levy [14], which goes for encoding of the worldwide design of an article and overlooking the points of interest. It is an arrangement of components acquired from Gabor wavelet

\section{Volume 4 Issue 12, December 2015}




\section{International Journal of Science and Research (IJSR) \\ ISSN (Online): 2319-7064}

Index Copernicus Value (2013): 6.14 | Impact Factor (2014): 5.611

separating on diverse introductions and patches, which is powerful in the progressions of scales, points, and brightening. The Gist features have accomplished great execution in article acknowledgment what's more, scene arrangement [15]-[17]. We research the utilization of Significance highlight for programmed cavity location so as to concentrate the neighborhood edge highlight and worldwide structure of cavities all the while, what's more, catch discriminative data for crater picture.

\section{Related Work}

J. R. Kim, J. P. Muller, and S. V. [3] propose that mechanized crater recognition calculation is exhibited which endeavors picture information. The calculation is quickly portrayed also, its application showed on an assortment of distinctive Martian geomorphologic regions and sensors (Viking Orbiter Camera, Mars Orbiter Camera (MOC), Mars Orbiter Laser Altimeter (MOLA), and High Resolution Stereo Camera (HRSC)).They indicate appraisal results through both an inter comparison of mechanized cavity areas with those from the physically inferred Mars Crater Consortium (MCC) inventory also, the physically inferred holes. The identification calculation achieves a precision of 70 to 90 percent and a quality component of 60 to 80 percent relying upon target sensor sort and geomorphology. They additionally introduce hole location results got from HRSC pictures locally available the ESA Mars Express on a correlation between physically decided Size-Frequency Distributions (SFDs) and those inferred completely naturally. The methodology portrayed seems to offer incredible potential for ordered examination, geometric and land examination and for different purposes of additional physical planetary surface mapping.

S. Liu, W. Ding, and T. F. Stepinski [8] consolidate dynamic learning with semi-regulated figuring out how to manufacture a new semi supervised dynamic class determination framework for crater location from high determination panchromatic planetary pictures. They propose the Semiregulated Active Class Selection Algorithm to iteratively enhance a unique little preparing set, without extra human marking exertion, to identify cavities from an expansive volume of pictures. They propose two methodologies to enhance location precision by coordinating characterization with investigation on unlabeled specimens. The Majority Vote Strategy is utilized to consequently acquire class marks by abusing unlabeled specimens from test pictures. In the same time, the Active Stability Strategy is utilized to acquire a fitting class dissemination in the built preparing set by identifying temperamental classes. By utilizing those two procedures, they effectively select test examples from test pictures into a current little introductory preparing set while re-taking in the classifier in the mean time. The proposed calculation is observationally assessed on an expansive testing Martian picture, displaying a vigorously cratered Martian landscape described by heterogeneous surface morphology. The exploratory results exhibit that the proposed approach accomplishes a higher exactness than other existing ways to deal with a vast degree.
D. Lowe [10] introduces a strategy for removing unmistakable invariant elements from pictures that can be utilized to perform solid coordinating between distinctive perspectives of an item or scene. The elements are invariant to picture scale and turn, and are appeared to give hearty coordinating over a considerable scope of relative mutilation, change in 3D perspective, expansion of commotion, and change in light. The elements are exceedingly unmistakable, as in a solitary element can be effectively coordinated with high likelihood against a vast database of components from numerous pictures. This paper likewise depicts a way to deal with utilizing these components for article acknowledgment. The acknowledgment continues by coordinating individual components to a database of components from known articles utilizing a quick closest neighbor calculation, trailed by a Hough Transform to distinguish bunches having a place with a solitary article, lastly performing check through slightest squares answer for predictable stance parameters. This way to deal with acknowledgment can heartily recognize objects among mess and impediment while accomplishing close ongoing execution.

R. Honda, Y. Iijima, and O. Konishi [11] study that a crater detection system for a large-scale image database is proposed. The original images are grouped according to spatial frequency patterns and both optimized parameter sets and noise reduction techniques used to identify candidate craters. False candidates are excluded using a self-organizing map (SOM) approach. The results show that despite the fact that a accurate classification is achievable using the proposed technique, future improvements in detection process of the system are needed.

S. Liu and. Ding, [12] proposes three procedures to enhance identification exactness by incorporating characterization with investigation on unlabeled specimens. The Majority Vote Strategy is utilized to consequently get class marks by misusing unlabelled examples. The De-Mixed Procedure is utilized on example separating to acquire dependable specimens. The Active Strength Strategy is utilized to acquire a suitable class circulation in the developed detecting so as to prepare set insecure classes. By utilizing those three systems, they effectively select test cases from test pictures into a current little introductory preparing set while revamping the classifier meanwhile. Their proposed calculations are observationally assessed on a vast high determination Martian picture, showing a vigorously cratered Martian territory described by heterogeneous surface morphology. The exploratory results show that the proposed methodology accomplishes a higher exactness than other existing ways to deal with a vast degree.

W. Ding and T. F. Stepinski [13] show an incorporated structure on auto detection of sub kilometer craters with boosting and exchange learning. The structure contains three key segments. To begin with, they use numerical morphology to proficiently recognize crater's applicants, the areas of a picture that can conceivably contain cavities. Just those districts possessing generally little partitions of the unique picture are the subjects of further preparing. Second, they concentrate and select picture composition highlights, in blend with regulated boosting outfit learning calculations, to

\section{Volume 4 Issue 12, December 2015}




\section{International Journal of Science and Research (IJSR) \\ ISSN (Online): 2319-7064}

Index Copernicus Value (2013): 6.14 | Impact Factor (2014): 5.611

precisely characterize crater's competitors into craters and non craters. Third, they coordinate move learning into boosting, to upgrade location execution in the locales where surface morphology contrasts from what is described by the preparation set. Their structure is assessed on an extensive test picture of $37,500 \times 56,250 \mathrm{~m} 2$ on Mars, which shows a vigorously cratered Martian landscape described by non uniform surface morphology. Experimental studies illustrate that the proposed crater discovery structure can accomplish a F1 score above 0.85 , a critical change over the other crater discovery calculations.

A. Oliva and A. Torralba, [15] propose a computational model of the acknowledgment of genuine scenes that sidesteps the division and the handling of individual items or locales. The methodology depends on a low dimensional representation of the scene, that they term the Spatial Envelope. They propose an arrangement of perceptual measurements (expectation, openness, harshness, development, toughness) that speak to the prevailing spatial structure of a scene. At that point, they demonstrate that these measurements may be dependably assessed utilizing phantom and coarsely restricted data. The model produces a multidimensional space in which scenes sharing enrollment in semantic classifications (e.g., avenues, roadways, and coasts) are anticipated shut together. The execution of the spatial envelope model demonstrates that particular data about article shape or character is not a necessity for scene arrangement and that displaying a comprehensive representation of the scene illuminates about its likely semantic classification.

A. Quattoni and A. Torralba [16], Indoor scene acknowledgment is a testing open issue in abnormal state vision. Most scene acknowledgment models that function admirably for open air scenes perform ineffectively in the indoor space. The fundamental trouble is that while some indoor scenes (e.g. hallways) can be very much described by worldwide spatial properties, others (e.g., book shops) are better portrayed by the articles they contain. All the for the most part, to address the indoor scenes acknowledgment issue they require a model that can misuse neighborhood and worldwide discriminative data. In this paper they propose a model based model that can effectively consolidate both wellsprings of data. To test our methodology they made a dataset of 67 indoor scenes classes (the biggest accessible) covering a extensive variety of areas. The outcomes demonstrate that their methodology can fundamentally beat a best in class classifier for the errand.
C. Siagian and L. Itti [17], depict and approve a basic context based scene acknowledgment calculation for versatile mechanical autonomy applications. The framework can separate open air scenes from different locales on a school grounds utilizing a multi scale set of ahead of schedule visual components, which catch the "significance" of the scene into a low-dimensional mark vector. Unmistakable from past methodologies, the calculation shows the favorable position of being organically conceivable and of having low computational many-sided quality, sharing its low-level elements with a model for visual consideration that may work simultaneously on a robot. They analyze characterization precision utilizing scenes taped at three open air locales on grounds (13,965 to 34,711 edges for every site). Isolating every site into nine fragments, they acquire portion grouping rates somewhere around $84.21 \%$ and $88.62 \%$. Joining scenes from all locales $(75,073$ edges altogether) yields $86.45 \%$ right characterization, exhibiting speculation and versatility of the methodology.

L. Bandeira, J. Saraiva, and P. Pina[19] presents an approach to recognize impact craters on the surface of Mars based on the analysis of a probability volume created by template matching.

S. Grigorescu, N. Petkov, and P. Kruizinga, [20] propose that surface elements that depend on the nearby power range acquired by a bank of Gabor channels are looked at. The components vary in the kind of nonlinear post-handling which is connected to the nearby power range. The accompanying elements are considered: Gabor vitality, complex minutes, and grinding cell administrator highlights. The ability of the comparing administrators to deliver particular element vector bunches for distinctive surfaces is analyzed utilizing two routines: the Fisher measure and the grouping result correlation. Both strategies give steady results. The grinding cell administrator gives the best separation and division results. The composition recognition capacities of the administrators what's more, their strength to non texture components is additionally looked at. The grinding cell administrator is the one and only that specifically reacts just to surface and does not give false reaction to non texture highlights for example, item shapes.

B. D. Bue and T. F. Stepinski, [22] present a novel methodology to crater discovery that uses advanced geology information rather than pictures. Craters are portrayed by topographic ebb and flow. Thresholding maps of shape changes topographic information into a parallel picture, from which craters are recognized utilizing a mix of division and discovery calculations. They apply their strategy in fact 


\section{International Journal of Science and Research (IJSR) \\ ISSN (Online): 2319-7064}

Index Copernicus Value (2013): 6.14 | Impact Factor (2014): 5.611

Table 1: Survey Table

\begin{tabular}{|c|c|c|c|c|}
\hline Paper Name & Author Name & Proposed Work & Advantages & Disadvantages \\
\hline $\begin{array}{c}\text { Automated Crater } \\
\text { Detection, A New Tool } \\
\text { for Mars Cartography } \\
\text { and Chronology }\end{array}$ & $\begin{array}{l}\text { J. R. Kim, J. P. } \\
\text { Muller, and S. } \\
\text { V }\end{array}$ & \begin{tabular}{|} 
Results through both an inter \\
comparison of automated crater \\
locations with those from the \\
manually-derived Mars Crater \\
Consortium (MCC) catalogue and the \\
manually-derived craters.
\end{tabular} & Good classification accuracy & $\begin{array}{c}\text { High computational } \\
\text { cost }\end{array}$ \\
\hline $\begin{array}{l}\text { Distinctive Image } \\
\text { Features from Scale- } \\
\text { Invariant Key points }\end{array}$ & D. Lowe & $\begin{array}{l}\text { Extracting distinctive invariant } \\
\text { features from images that can be used } \\
\text { to perform reliable matching between } \\
\text { different views of an object or scene. }\end{array}$ & $\begin{array}{c}\text { more efficient feature } \\
\text { extraction and the ability to } \\
\text { identify larger } \\
\text { numbers of features }\end{array}$ & $\begin{array}{l}\text { A much higher } \\
\text { computational cost }\end{array}$ \\
\hline $\begin{array}{l}\text { Sub kilometer Crater } \\
\text { Discovery with } \\
\text { Boosting and Transfer } \\
\text { Learning } \\
\text { Systems }\end{array}$ & \begin{tabular}{|} 
W. Ding and T. \\
F. Stepinski
\end{tabular} & $\begin{array}{c}\text { An integrated framework on auto } \\
\text { detection of sub kilometer craters with } \\
\text { boosting and transfer learning }\end{array}$ & $\begin{array}{l}\text { great potential to produce } \\
\text { surveys of small craters over } \\
\text { entire surfaces of planets }\end{array}$ & $\begin{array}{l}\text { decreases and/or } \\
\text { disappears when } \\
\text { classifying crater } \\
\text { candidates that are } \\
\text { less similar to those in } \\
\text { the training set }\end{array}$ \\
\hline $\begin{array}{l}\text { Modeling the Shape of } \\
\text { the Scene: A Holistic } \\
\text { Representation of the } \\
\text { Spatial Envelope }\end{array}$ & $\begin{array}{l}\text { A. Oliva and A. } \\
\text { Torralba }\end{array}$ & $\begin{array}{l}\text { Computational model of the } \\
\text { recognition of real world scenes that } \\
\text { bypasses the segmentation and the } \\
\text { processing of individual objects or } \\
\text { regions }\end{array}$ & $\begin{array}{c}\text { possibility to generate a } \\
\text { meaningful description of the } \\
\text { space }\end{array}$ & $\begin{array}{l}\text { Primarily for man- } \\
\text { made vs. natural } \\
\text { differences }\end{array}$ \\
\hline $\begin{array}{l}\text { Rapid Biologically- } \\
\text { Inspired Scene } \\
\text { Classification Using } \\
\text { Features Shared with } \\
\text { Visual Attention }\end{array}$ & $\begin{array}{l}\text { C. Siagian and } \\
\text { L. Itti }\end{array}$ & $\begin{array}{l}\text { simple context based scene } \\
\text { recognition algorithm for mobile } \\
\text { robotics applications }\end{array}$ & \begin{tabular}{|} 
The advantage of being \\
biologically plausible and of \\
having low computational \\
complexity, sharing its low- \\
level features with a model for \\
visual attention that may \\
operate concurrently on a \\
robot
\end{tabular} & $\begin{array}{l}\text { Does not uses bottom- } \\
\text { up salient cues to } \\
\text { produce a useful } \\
\text { topographical map for } \\
\text { navigation }\end{array}$ \\
\hline
\end{tabular}

requesting test site and contrast the outcomes with the current inventory of physically recognized cavities. Their calculation finds numerous little craters not recorded in the manual inventory; however it neglects to distinguish intensely debased craters. A definite quality evaluation of the calculation is introduced. The geography based crater recognition calculation offers a generally basic and prepared to-utilize device for ID and portrayal of crisp effect crater with a sufficient execution for the quick application to Martian geomorphology.

\section{Conclusion}

In this paper we conclude that there are many methods for crater detection. There are some manual methods for crater detection but these methods took more time for detection of crater. Also, there are different types of methods for crater detection which are based on automatic crater detection. So these methods are easy $\&$ less time consuming methods for crater detection than manually identifying crater.

\section{References}

[1] P. G. Wetzler and R. Honda, "Learning to detect small impact craters," in Proc. 7th IEEE Workshops Appl. Comput. Vis. (WACV/MOTION), 2005,pp. 178-184.
[2] J. Yin, Y. Xu, and H. Li, "A novel method of crater detection on digital elevation models," in Proc. IEEE Conf. 33rd Geosci. Remote Sens. Symp.(IGARSS), Melbourne, V.I.C., Australia, 2013, pp. 4150-4153.

[3] J. R. Kim, J. P. Muller, and S. V. Gasselt, "Automated crater detection, a new tool for mars cartography and chronology," Photogramm. Eng.Remote Sens., vol. 71, no. 10, pp. 1205-1217, Oct. 2005.

[4] L. Bandeira, W. Ding, and T. F. Stepinski, "Detection of sub-kilometer craters in high resolution planetary images using shape and texture features," Adv. Space Res., vol. 49, no. 1, pp. 64-74, Jan. 2012.

[5] H. Li and J. Yin, "Crater detection based on local nonnegative matrix factorization," in Proc. IEEE Conf. 33rd Geosci. Remote Sens. Symp. (IGARSS), QC, Canada, 2014, pp. 3050-3053.

[6] Y.Mu,W. Ding, D. Tao, and T. F. Stepinski, "Biologically inspired model for crater detection," in Proc. Int. Joint Conf. Neural Netw., San Jose, CA,USA, 2011, pp. 2487-2494.

[7] R. Martins, P. Pina, J. S. Marques, and M. Silveira, "Crater detection by a boosting approach," IEEE Geosci. Remote Sens. Lett., vol. 6, no. 1,pp. 127-131, Jan. 2009.

[8] S. Liu, W. Ding, and T. F. Stepinski, "Semi-supervised based active class selection for automatic identification of sub-kilometer craters," in 7th Int.Symp. Image Signal Process. Anal., Dubrovnik, Croatia, 2011, pp. 615-620. 


\section{International Journal of Science and Research (IJSR) \\ ISSN (Online): 2319-7064}

Index Copernicus Value (2013): 6.14 | Impact Factor (2014): 5.611

[9] A. Liu and W. Pan, "Crater detection algorithm with part PHOG features for safe landing," in Proc. Int. Conf. Syst. Informat., Yantai, China, 2012,pp. 103-106.

[10]D. Lowe, "Distinctive image features from scaleinvariant key points," Int.J. Comput. Vis., vol. 60, no. 2, pp. 91-10, Nov. 2004.

[11]R. Honda, Y. Iijima, and O. Konishi, "Mining of topographic feature from heterogeneous imagery and its application to lunar craters," Prog.Discovery Sci., vol. 2281, pp. 395-407, 2002

[12] S. Liu and. Ding, "Adaptive selective learning for automatic identification of sub-kilometer craters," Neurocomputing, vol. 92, pp. 78-87, Sep.2012.

[13] W. Ding and T. F. Stepinski, "Sub-kilometer crater discovery with boosting and transfer learning," ACM Trans. Intell. Syst. Technol., vol. 2,pp. 1-22, Jul. 2011.

[14] M. C. Potter and E. I. Levy, "Recognition memory for a rapid sequence of pictures," J. Exp. Psychol., vol. 81, no. 1, pp. 10-15, Jul. 1969.

[15] A. Oliva and A. Torralba, "Modeling the shape of the scene: A holistic representation of the spatial envelope," Int. J. Comput. Vis., vol. 42, no. 3,pp. 145-175, May 2001.

[16]A. Quattoni and A. Torralba, "Recognition indoor scenes," in Proc. IEEEConf. Comput. Vis. Pattern Recogn. (CVPR), Miami, FL, USA, 2009, pp.413-420.

[17] C. Siagian and L. Itti, "Rapid biologically-inspired scene classification using features shared with visual attention," IEEE Trans. Pattern Anal.Mach. Intell., vol. 29, pp. 300-312, Feb. 2007.

[18] G. Salamuniccar and S. Loncaric, "Method for crater detection from Martian digital topography data using gradient value/orientation, morphometry,vote analysis, slip tuning, and calibration," IEEE Trans.Geosci. Remote Sens., vol. 48, no. 5, pp. 2317-2329, May 2010.

[19] L. Bandeira, J. Saraiva, and P. Pina, "Impact crater recognition on Mars based on a probability volume created by template matching,"IEEE Trans. Geosci. Remote Sens., vol. 45, no. 12, pp. 4008-4015,Dec. 2007.

[20] S. Grigorescu, N. Petkov, and P. Kruizinga, "Comparison of texture features based on Gabor filters," IEEE Trans. Image Process., vol. 11, no. 10,pp. 11601167, Oct. 2002.

[21] A. Oliva and A. Torralba, "Building the gist of a scene: The role of global image feature in recognition," Prog. Brain Res. Vis. Percept., vol. 155,pp. 23-36, 2006.

[22] B. D. Bue and T. F. Stepinski, "Machine detection of Martian impact craters from digital topography data," IEEE Trans. Geosci. Remote Sens.,vol. 45, no. 1, Jan. 2007.

[23] G. Troglio and J. L.Moigne, "Automatic extraction of ellipsoidal features for planetary image registration," IEEE Geosci. Remote Sens. Lett., vol. 9, no. 1, Jan. 2012.

[24] L. Breiman, "Random forests," Mach. Learn. J., vol. 45, no. 1, pp. 5-32,Oct. 2001.

[25] V. Lepetit and P. Fua, "Keypoint recognition using randomized trees,'IEEE Pattern Recogn. Mach. Intell., vol. 28, no. 9, pp. 1465-1479, Sep.2006.

\section{Author Profile}

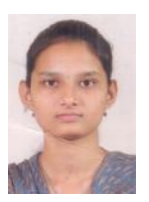

Jyoti Patil, is currently pursuing M.E (Computer Engineering) From Department of Computer Engineering, Jayawantrao Sawant College of Engineering, Hadapsar, affiliated to Savitribai Phule, Pune University, Pune, Maharashtra, India -411028. She received her B.E. (Computer Engineering) Degree from MKSSS Cummins College of Engineering For Women, affiliated to Savitribai Phule Pune University, Pune, Maharashtra, India - 411007. Her area of interest is Image Processing, Data Mining \& Networking.

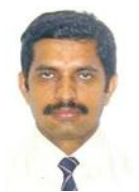

Prof. Dr. Srinivas Narasimha Kini, received his $\mathrm{PhD}$ Degree from Cochin University of Science and Technology, Thrikkakara, South Kalamasserry, Cochin. He received his M.E (Computer Science and Engineering) Degree from B.M.S. College of Engineering, Basavanagudi, Bangalore, India. He received his B.E (Computer Science and Engineering) Degree from K L E Society's College of Engineering Udyambaug Belgaum, India. He is currently working as Professor at Department of Computer Engineering, Jayawantrao Sawant College of Engineering, Hadapsar, Pune, India 411028, affiliated to Savitribai Phule Pune University, Pune, Maharashtra, India -411007. His area of interest is Image Processing, Distributed Computing System, Network Security, Data Mining etc. 